shown elsewhere that old age, poor survival, DHA of residence, and place of death were all positively associated with DCO registration. ${ }^{3}$ All the studies Gulliford cites have used the Thames cancer registry as the sampling frame for studies of case notes and all exclude DCO cases from their sample. Our second concern relates to the use of cancer registry data for health services research. National Health Service (NHS) purchasers are now funding registries directly; we consider it essential that the reliability and the validity of registry data be confirmed before these are used as a basis for needs assessment, service outcomes, and provision. Registry data cannot be used when they are inaccurate or incomplete. In a previous paper we have shown that part of the problem lies with the incompleteness of clinical notes, ${ }^{9}$ and in that paper we chose to focus on quality control within the registry.

Gulliford asks how we assessed the reliability of our own data abstraction. Before beginning work, the two doctors who carried out the abstraction liaised with registry staff to confirm the criteria used when coding date of diagnosis, stage, and treatment. Data were abstracted on each set of case notes by the two doctors separately and checked for interobserver bias. Further checks took place at clinical audit meetings with surgeons and pathologists when cases with absent or discordant information was audited. All of this is described in a previous work cited in our paper. $^{9}$

We regret the ambiguity in the abstract which could be taken to mean that the registry had data on treatment for DCO cases. However, the background and methods sections make clear that the registry definition of DCO cases was the one followed in this study. References to disagreements involving DCO cases in the abstract would be better described as disagreements involving DCO cases for which we subsequently retrieved clinical data. DCO cases are important because their exclusion from the sample can bias measurements of treatment and survival. In other papers we have attempted to measure the impact of DCO cases on national survival and the effect of losing them. ${ }^{3} 10$

In conclusion may we identify what we take to be the strengths of our paper? Our objective was to identify factors in the registration process affecting reliability. We showed error in three areas in which the registry has explicit written policies: the retrospective follow up of DCO cases, six-month active follow up of cases, and the coding criteria for date of diagnosis. The registry has responded positively to this audit and to our recommendations for improving the internal quality of registry data.

ALLYSON M POLLOCK NEIL VICKERS
Department of Public Health Sciences, Department of Public Health Sciences,
St George's Hospital Medical School, Cranmer Terrace,
London SW17 ORE

1 Pollock AM, Vickers N. Reliability of Thames cancer registry data on 673 cases of colorectal cancer: effect of the registration process. Quality in Health Care 1995;4:184-9.
2 Gulliford MC, Bell J, Bourne HM, Petruckevitch A. The reliability of Cancer registry records. British $\mathcal{f}$ Cancer 1993;67: 819-21.

3 Pollock AM, Vickers N. Why are a quarter of all cancers in south east England registered by "death certificate only" (DCO)? Factors related to DCO registrations in the Thames Cancer Registry between 1987 and $1989 . B r$ 7 Cancer 1995;71:637-41.

4 Thames Cancer Registry. Cancer in South East Thames 1987-9. Sutton, Surrey: Thames Cancer Registry, 1992.

5 Thames Cancer Registry. Cancer in South West Thames 1987-9. Sutton, Surrey: Thames Thames 1987-9. Sutton,

6 West RR. Accuracy of cancer registration. $B r \mathcal{F}$ Prev Soc Med 1976;30:187-92.

7 Lapham R, Waugh NR. An audit of the quality of cancer registration data. $\mathrm{Br} \mathcal{F}$ Cancer 1992;66:552-4.

8 Chow WH, Devesa SS. Death certificate reporting of colon and rectal cancers. $\mathscr{F} A M A$ 1992;267:3028.

9 Vickers N, Pollock A. Incompleteness and retrieval of case notes in a case note audit of colorectal cancer. Quality in Health Care 1993;2:170-4.

10 Pollock A M, Vickers N. The impact on colorectal cancer survival of cases registered by "death certificate only" - implications for
national survival data. Br $f$ Cancer 1994; 70:1229-31.

\section{Medication errors during hospital drug rounds}

In their paper Ridge et $a l^{1}$ set out to find the nature and rate of drugs given in error in one National Health Service (NHS) hospital. It is important to distinguish between the authors' focus, which was errors that occurred at the time of the nurse giving the drug, and prescribing errors that originate with the doctor and already exist on the prescription. Prescribing errors were not examined by the authors as their survey recorded only those errors that could be classed as deviations from the doctor's medication order as written on the patient's chart. ${ }^{2}$

Although it is important that hospitals do review the effectiveness of their current drug supply and administration systems (as the authors suggest), it is incorrect to support the seriousness of this argument with reference to a review of coroner's records which concluded that about a fifth of deaths relating to prescribing and giving drugs were due to errors. ${ }^{3}$

In this review, a total of 3277 deaths came to inquest $(3.8 \%$ of all deaths in the years 1986-91) and the review of coroner's cases actually identified 46 relevant deaths (due to adverse drug reactions or errors in prescribing or giving drugs). Of these 46 , death was attributed to errors in medication in 10 cases, with an even mix of primary and secondary care cases, but of these 10 most were due to prescribing errors with possibly only one death due to a nurse giving a drug in error (and that involved oxygen).

The overall risk of death due to errors or adverse drug reactions was judged to be very small - about one in 2000 of all deaths during the study period, and of course, unlike in the paper by Ridge et al, there was no baseline for the number of total events that were potentially adverse that is, the number of doses of medicines prescribed and given during the six year period.
MICHAEL WILCOCK

Cornwall and Isles of Scilly Health Authorities, Health Authorities,
fohn Keay House, St Austell,

Cornwall PL25 4Df

1 Ridge KW, Jenkins DB, Noyce PR, Barber ND. Medication errors during hospital drug rounds. Quality in Health Care 1995;4: 240-3.

2 Allan EL, Barker KN. Fundamentals of medication error research. Am $\mathcal{f}$ Hosp Pharm 1990;47:555-71.

3 Ferner RE, Whittington RM. Coroner's cases of death due to errors in prescribing or giving medicines or to adverse drug reactions: Birmingham 1986-91. F $R$ Soc Med 1994; 87:145-8.

\section{Preprinted assessment sheet}

Goodyear and Lloyd pointed out the advantages of a preprinted assessment sheet, ${ }^{1}$ but I would like to point out the danger of implementing this method in the hospital setup, specially for junior doctors in training.

Good history taking in medicine has for generations been the main method of educating medical students and junior doctors. Full evaluation of the history of a patient's complaints is crucial to making a correct diagnosis, and helps in planning the management. Every doctor spends the rest of his or her professional life relearning the lesson. The doctor's first task is to listen and observe, not only to obtain information about the current problem but also to understand the patient as a person and to learn about their life situation. ${ }^{2}$

Symptoms identified by taking a history provide some of the most important items of information used in the process of diagnosing a disease. When patients describe the symptoms for which they are seeking professional attention, they are also reporting the story of an illness as they have lived, and remembered it, and so it can vary. To some extent, symptoms are universal human experience. Virtually every person experiences some discomfort for which he or she is seeking some help.

Talking with a patient has a third function: it helps that person to feel that he or she is understood, and it thereby helps to establish a therapeutic relation. A style of questioning narrowly shaped for the sole purpose of diagnosing a disease ignores much of what patients have experienced and many of their concerns and questions. It therefore often prevents the development of a trusting relation, and diminishes the chances of helping the patient. Talking with a patient about the experience of being ill, on the other hand, can have great value even when nothing can be done about the disease. ${ }^{3}$

Collecting information with a preprinted assessment sheet, or computer may be good, and may be more efficient, but is not advisable for young doctors in training. It is the duty of the senior experienced doctors to identify deficiencies in history taking by a junior doctor, and help him or her to rectify the deficiencies and thus help them to be good clinicians.

The disadvantage of a preprinted assessment sheet is that you forget to 
think of relevant questions and the sequence of questioning required to arrive at a diagnosis. This may result in the future clinician learning to fill in the blanks meticulously, but unable to collect, and analyse data in their brain. This type of "fill in the blank questionnaire" requires no special history taking skill, and may also result in delay in arriving at diagnosis in an emergency situation.

I have come across some junior doctors meticulously trying to take a history with these preprinted sheets, when they should have concentrated on treating the patient. The patient is uncomfortable, and at times very ill, but the doctor starts asking questions, as printed, and fails to recognise the patient's problem, nor give importance to what the patient wants to say. This makes the patient feel that the doctor is not keen on understanding his current problem, and so loses confidence in the management. The other problem is that when the doctor is taking a history with a preprinted history sheet there is no eye contact with the patient because the doctor is keen on reading the printed questions. This breaks the patient-doctor relation and can be disadvantageous to both doctor and patient. Let us not forget that today's junior doctors are our physicians and surgeons tomorrow.

\section{KADIYALI M SRIVATSA Department of Paediatrics, Stoke Mandeville Hospital, Aylesbury, Bucks HP21 $8 A L$}

1 Goodyear HM, Lloyd BW. Can admission notes be improved by using pre-printed assessment sh

2 Edwards CRW. History and general principles governing the physical examination. In Macleod's clinical examination, 8th ed. Edinburgh, Churchill Livingstone, 1990.

3 Bates B, Hoekelman RA. Interviewing and history taking. In: Guide to physical examination and history taking sth ed. Philadelphia: JB Lippincott, 1990.

AUTHOR'S REPLY - We thoroughly agree with Srivatsa's view that taking a history is about much more than collecting information. We agree that taking a history is also a powerful way of building a relation with the patient and that this process may be jeopardised by the act of writing the history down. Srivatsa argues that the use of preprinted prompts to record the history is more likely to jeopardise the process of building a relation than traditional ways of recording the history. Srivatsa also argues that the use of preprinted prompts may lead to doctors failing to use their brains to collect and analyse data and that this can lead to delays in diagnosis.

Our study did not specifically study either of these points but we do not think they are valid. We teach our juniors to ask the patient (or parent) "What is the problem?" and then to listen. Only when the patient has had his or her say should the doctor write anything. What is then written is the "presenting complaint" and the start of the "history of the presenting complaint." We then ask the trainee to complete the rest of the history of the presenting complaint in the traditional way. The first page of our notes is dedicated to the history of the presenting complaint and is blank except for four prompts at the bottom (about medication, food, fluids, and activity).

Although there are repeated prompts in the rest of the history and examination we emphasise to our trainees the importance of writing appropriate notes in the blank space surrounding each prompt. In our paper we reported that there were fewer words written in notes with preprinted prompts. Thus we think that far from alienating patients the use of notes with preprinted prompts improves rapport because they are quicker to complete.

We also reject Srivatsa's worry that diagnosis may sometimes be delayed because trainees will blindly tick boxes rather than use their brains to record the history. As already emphasised recording the history of the presenting complaint (the most important element of the history) is done in the traditional way. Furthermore all of our prompts concern what members of the department considered were "core clinical details" that should be recorded in all cases. Our study showed that the use of notes with preprinted prompts meant that almost invariably these details were recorded. It is hard to see how recording this information could lead to delays in diagnosis.

HELEN M GOODYEAR BW LLOYD Children's Department North Middlesex Hospital, Sterling Way, London N18 $1 Q X$

\section{SOFTWARE REVIEW}

Pinpoint for windows. ( $£ 299$ plus VAT; site licence $£ 750$ plus VAT (three users). Cambridge: Longman Logotron, 1993.

One aspect of improving quality in health care is using medical and clinical audit effectively to bring about change. Doctors, nurses, and other paramedical groups are increasingly required to spend time auditing their current practices and clinical processes. It is helpful to be able to design a data entry form or questionnaire from which information can be entered on to a computerised database used to sort, categorise, and summarise this information. Many database packages are now available which can be customised to perform these tasks, but they are often difficult to teach to newcomers to computing. The program used in this hospital, Pinpoint for Windows, is ideal for carrying out audit projects.

Pinpoint is a menu driven information collection and analysis package designed with market research and customer surveys in mind, which requires no prior knowledge of programming. The program requires an IBM compatible personal computer (80286 or higher processor, at least $2 \mathrm{MB}$ of $\mathrm{RAM}$, and at least $3 \mathrm{MB}$ of free hard disk space) and runs using the Microsft Windows (version $3 \cdot 1$ onwards) graphical interface with a mouse. It comes with an excellent manual.

The first task - creating a data entry form or questionnaire - is fairly easy as you first decide where to place the question of a blank form and Pinpoint then provides a dialogue box from which the question (or field) can be customised into field type. Field types include: (a) fixed length text, (b) free form text (for example, to record comments, (c) numerical, (d) date, (e) yes/no, ( $f$ ) multiple choice, and $(g)$ ordered choice.

Once the field type has been chosen Pinpoint automatically provides check boxes for answers. The question and answer areas can be moved around the form and embellished with free standing text, drawing boxes, and lines to make attractive looking data collection forms. Forms can be modified (for example, with an additional question) at any stage, and Pinpoint will automatically update all previously completed answer sheets. This is useful when additional information is requested to be collected during the course of an audit.

Photocopies of the data entry sheet can be used for data collection. The information may then be transformed on to a similar form on the computer (the answer sheet) Pinpoint can be used to analyse the data and present them as summaries or graphs. The data can then be sorted, selections made for subgroup analysis, and categorised (for example, answers from Yes/No or multiple choice questions) and calculations performed. The information can be summarised using descriptive statistics, frequency, and

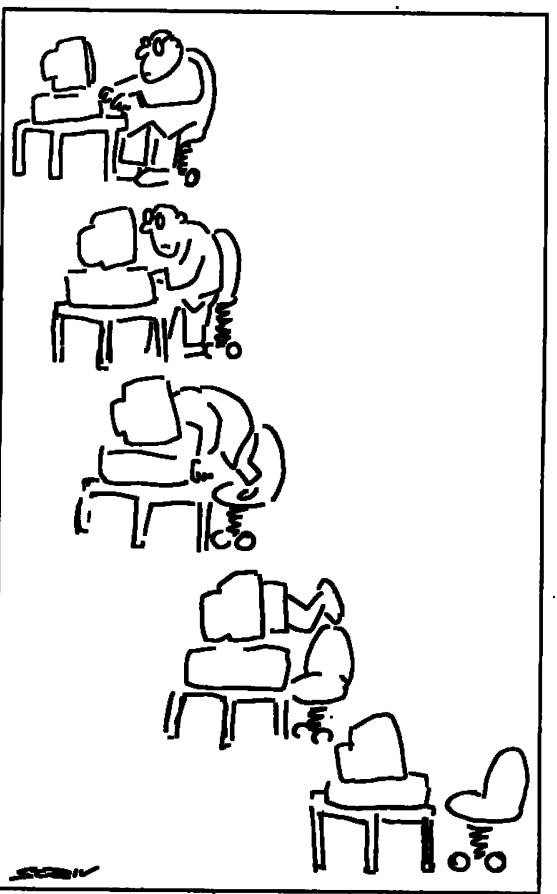

From now on we are electronic! 